\title{
Supporting information: Nanoelectromechanical tuning of dual-mode resonant dielectric metasurfaces for dynamic amplitude and phase modulation
}

\author{
Hyounghan Kwon, ${ }^{1,2,3}$ Tianzhe Zheng, ${ }^{1,3}$ and Andrei Faraon ${ }^{1,2, *}$ \\ ${ }^{1}$ T. J. Watson Laboratory of Applied Physics and Kavli Nanoscience Institute, \\ California Institute of Technology, 1200 E. California Blvd., Pasadena, CA 91125, USA \\ ${ }^{2}$ Department of Electrical Engineering, California Institute of Technology, \\ 1200 E. California Blvd., Pasadena, CA 91125, USA \\ ${ }^{3}$ These authors contributed equally
}

* Corresponding author: A.F.: faraon@ caltech.edu 


\section{MEASUREMENT PROCEDURE}

All of the reflection spectra presented in this paper are characterized using the set-ups shown schematically in Figure S1 [1]. A tunable laser (Photonetics, TUNICS-Plus) is used as the light source and the wavelength of the light is tuned from $1450 \mathrm{~nm}$ to $1580 \mathrm{~nm}$. We use a beam splitter in front of the fiber collimator (Thorlabs, F260FC-1550) to capture the power from the source and send the light to the sample. For reference, the power from the source is captured by a InGaAs detector (Thorlabs, PDA10CS). Due to variation in polarization states from the laser, a quarter waveplate in front of a polarization beamsplitter (PBS) is used to prevent low transmission through PBS at specific wavelengths, increasing signal-to-noise ratio over the entire spectrum. The PBS, a half waveplate (HWP), and a polarizer are inserted to set the polarized state of the incident light to TE polarization. The sample at the object plane is imaged by a $20 \times$ infinity-corrected objective lens (Mitutoyo, M Plan Apo NIR) and a tube lens with a focal length of $200 \mathrm{~mm}$. As the tube lens and the objective lens are forming a 4-f system, the movement of the tube lens in $x$-axis enables to adjust the angle of illuminated light. At the image plane, a pinhole with a diameter of 400 $\mu \mathrm{m}$ is inserted to select a region of interest with a diameter of $20 \mu \mathrm{m}$ in the object plane. The spatially filtered light was either focused onto another InGaAs detector for the measurement of the spectra, or imaged on an InGaAs SWIR camera (Goodrich, SU320HX-1.7RT) using relay optics. All spectra in this paper were obtained by dividing the signal from the sample by the signal from the sources. Due to different input polarization states, the incidence power onto the sample varies in different wavelengths. Thus, the spectra are further normalized by the spectra from the gold electrode. For the measurement of dynamic responses shown in Figs. 3-5, bias voltages, both DC and AC, are applied with a function generator (FeelTech, FY6600-60M).

To measure the phase response shown in Fig. 5d, we use a Michelson-type interferometer setup. A part of the setup marked by a black dashed box in Figure S1 is only utilized for the phase measurement. Specifically, the reference beam interferes with the reflected beam of the sample and forms fringe patterns at both image and camera planes enabling the measurement of reflected phase as a function of applied biases from $0 \mathrm{~V}$ to $4 \mathrm{~V}$. As shown in Figures S5b and S5C, the fringes are not shifted on the electrode but on the gratings under different external biases. The phase responses under the external biases are calculated by using the corresponding shifts of the fringes

on the grating shown in Figure S5c. We used the formula $2 \pi \frac{\delta w}{w_{0}}$ to calculate the phase shifts, where $\delta w$ is the averaged shift of the fringes in the unit of camera pixel number, and $w_{0}$ is the pixel 
number of a period of the fringe. The measured $w_{0}$ is 10 camera pixels. Finally, the phase shifts are averaged from all five pictures in Figure S5c and plotted in Fig. 5d. 


\begin{tabular}{|c|c|c|c|c|}
\hline Figure & $21(\mathrm{~nm})$ & $\mathrm{w}(\mathrm{nm})$ & $\mathrm{g}_{1}(\mathrm{~nm})$ & $\mathrm{g}_{2}(\mathrm{~nm})$ \\
\hline Figures $3 b, 3 d$, and 4 & 1400 & 495 & 245 & 165 \\
\hline Figures $3 c$ and $3 e$ & 1320 & 495 & 185 & 145 \\
\hline Figure 5 & 1332 & 494 & 144 & 200 \\
\hline Figure S2a & 1400 & 460 & 240 & 240 \\
\hline Figure S2b & 1400 & 480 & 220 & 220 \\
\hline
\end{tabular}

Table S1 Design parameters for all nanomechanical gratings in this work. $2 l$ : Lattice constant for a pair of the silicon bars. $w$ : Width of the silicon bar. $g_{1}$ : Gap between the silicon bars having voltage difference. $g_{2}$ : Gap between the silicon bars having same voltage. 


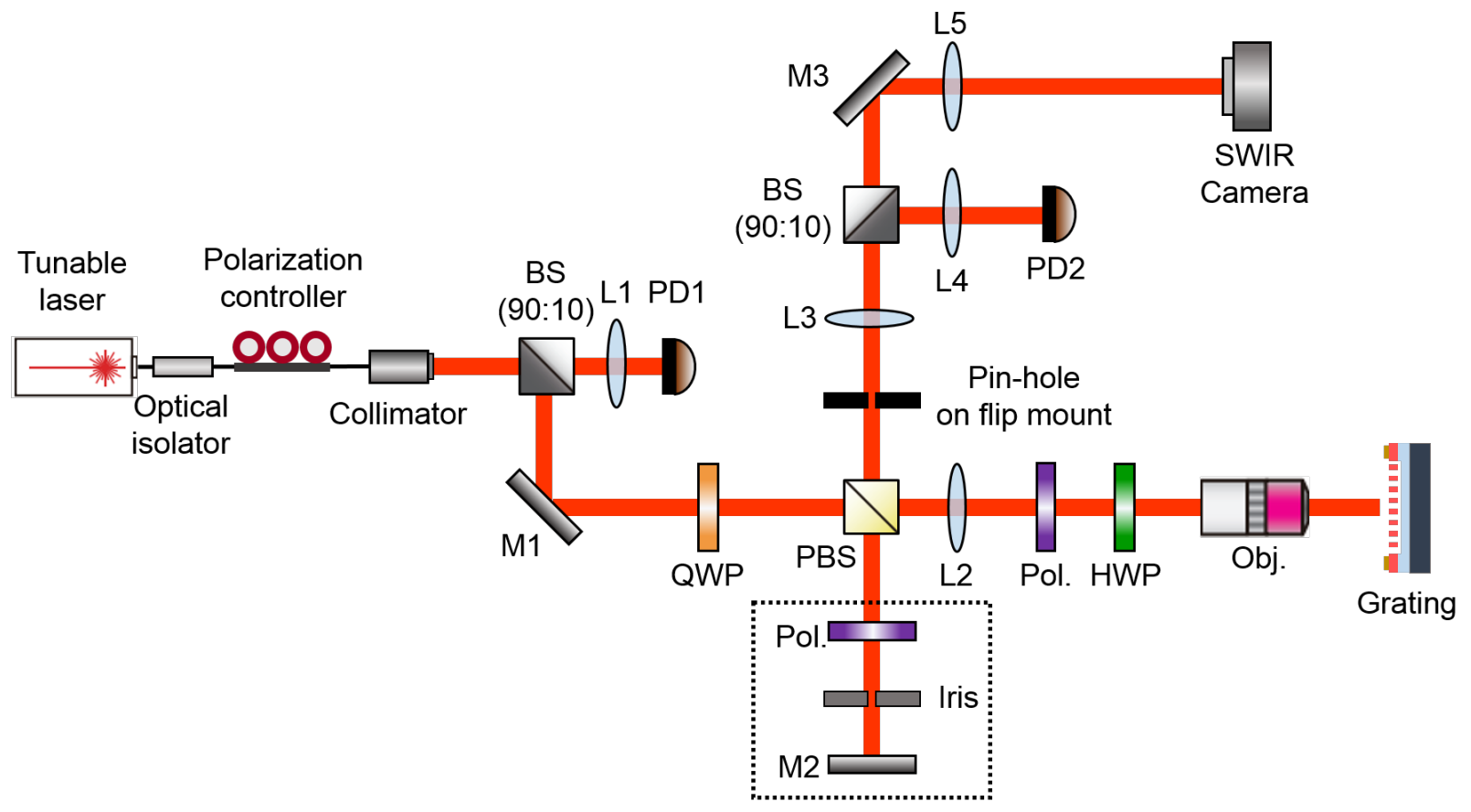

Figure S1 Schematic illustration of the experimental setup. Red lines represent the paths of the light. To achieve the reflection spectra of the TE-polarized input light, Pol. and HWP in front of the objective lens are aligned to 45 and 67.5 degree, respectively. A black dashed box represents optical elements exploited to generate reference beam for the phase measurement shown in Fig. $5 \mathbf{d}$ of the main text and Figure S5. Pol.: linear polarizer. BS: beamsplitter. PBS: polarizing beamsplitter. L: lens. PD: photodetector. M: mirror. QWP: quarter waveplate. HWP: half waveplate. Obj.: microscope objective lens. SWIR camera: short-wave infrared camera 

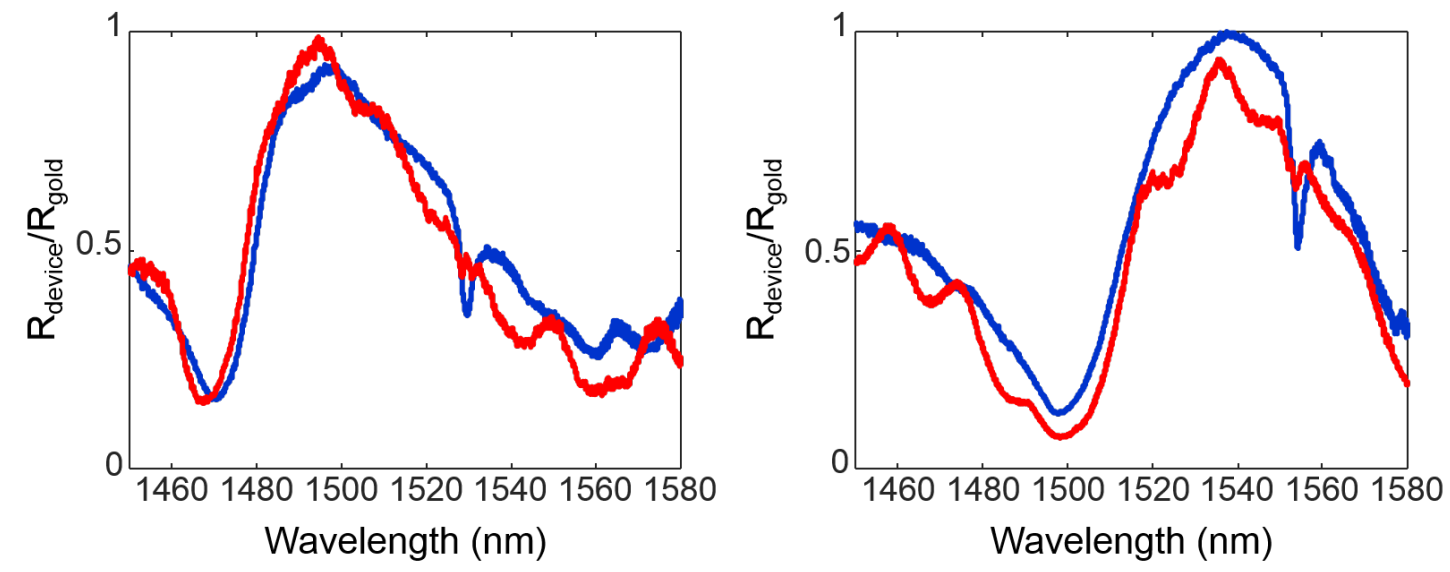

Figure S2 Measurement of angle-sensitive reflection spectra. a and $\mathbf{b}$ Red and blue curves show the reflection spectra for normal and 6 degree tilted TE polarized incident light, respectively. With $500 \mathrm{~nm}$ thickness and $700 \mathrm{~nm}$ period of the lattice, the design parameters of a and $\mathrm{b}$ are shown in Table S1. 


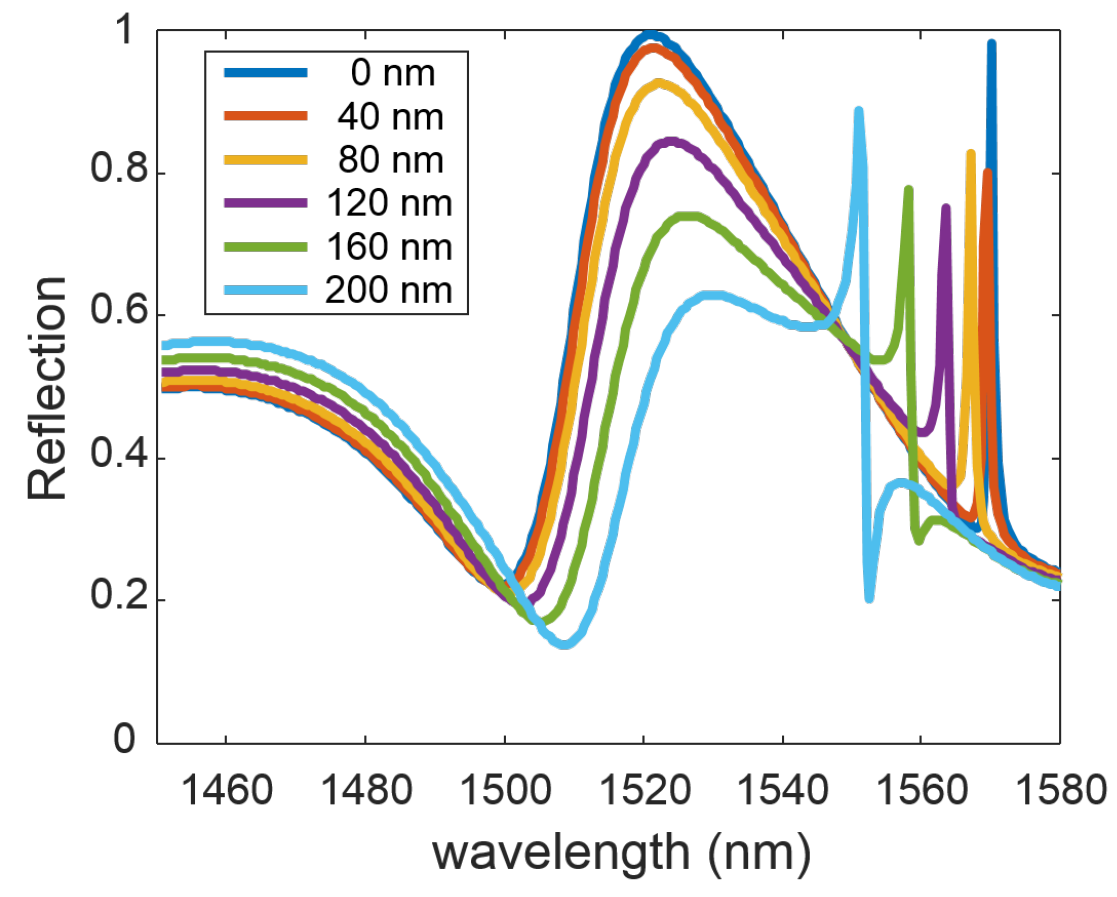

Figure S3 Numerical investigation related to spectral shifts of the resonances induced by actuation. The spectra are simulated under six different values of $g_{2}-g_{1}$ and plotted in different colors. The value of $g_{2}-g_{1}$ for each color is shown in legends. The width and lattice constant of the device are $480 \mathrm{~nm}$ and 700 $\mathrm{nm}$, respectively. 


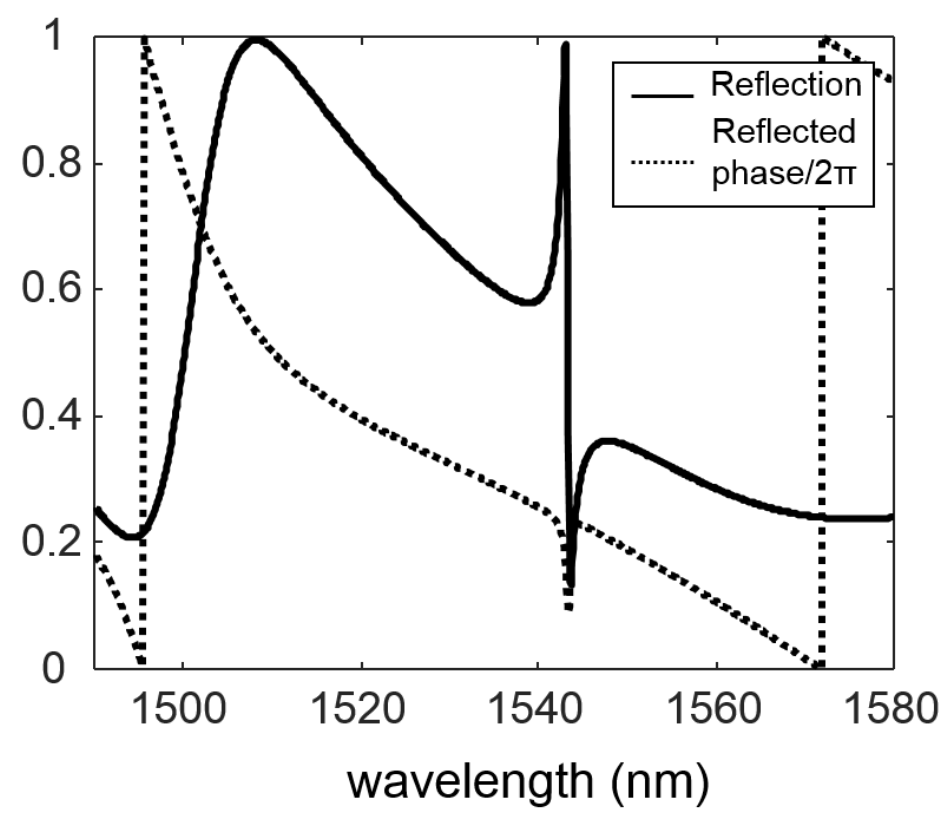

Figure S4 Calculated reflection and reflected phase spectra for single BIC resonance. The quasi-BIC mode is apart from the peak of the GMR. At the resonance wavelength of $1543 \mathrm{~nm}$, the calculated phase response of the quasi-BIC mode is smaller than $55 \mathrm{deg}$. The width and the lattice of the structures are 475 $\mathrm{nm}$ and $666 \mathrm{~nm}$, respectively. 
a

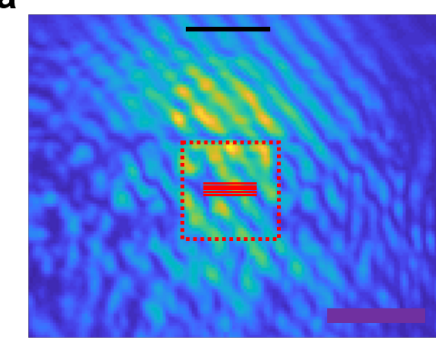

b

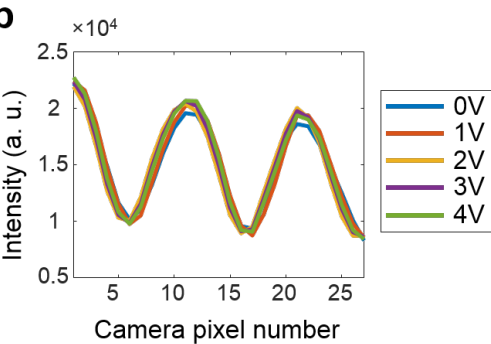

C
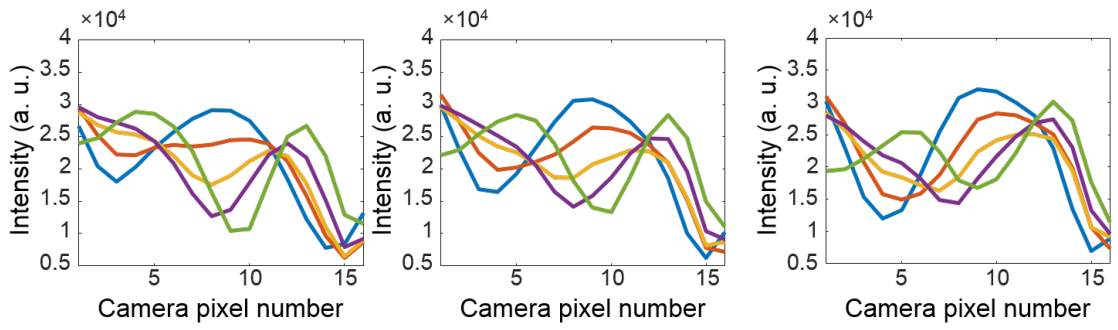
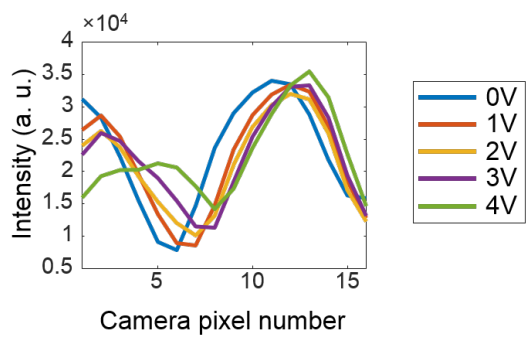

Figure S5 Fringe analysis for phase response measurement. a A camera image of the fringes on the measured device. The red dashed square shows the position of the measured device. Five solid red lines inside the red square and a black solid line show the places that we use to analyze the phase responses on the grating and the electrode, respectively. Scale bar denotes $30 \mu \mathrm{m}$. b The fringe patterns on the electrode under the external biases from $0 \mathrm{~V}$ to $4 \mathrm{~V}$. The fringe patterns are captured along the black solid line in a. The fringes are nearly identical and not shifted for the different voltages. $\mathbf{c}$ The five fringe patterns on the grating under the external biases from $0 \mathrm{~V}$ to $4 \mathrm{~V}$. The fringe patterns are captured at the center of the grating along the five red solid line in a. The five plots show the clear shifts of the fringes. In $\mathrm{b}$ and $\mathrm{c}, x$ and $y$ - axes are pixel number and pixel value of the camera, respectively. The applied biases for all colors are denoted in outsets in $\mathrm{b}$ and $\mathrm{c}$. 


\section{REFERENCES}

[1] Horie, Y., Arbabi, A., Arbabi, E., Kamali, S. M. \& Faraon, A. High-speed, phase-dominant spatial light modulation with silicon-based active resonant antennas. ACS Photonics 5, 1711-1717 (2017). 